\title{
Characterization of the Higgs boson candidate at CMS
}

\author{
Marco Zanetti ${ }^{1, a}$ \\ ${ }^{1}$ MIT Laboratory for Nuclear Science, 77 Massachusetts Avenue,Cambridge, MA 02139
}

\begin{abstract}
The first studies performed by the CMS collaborations of the properties of the recently discovered Higgs boson candidate are presented. The data sample considered corresponds to the integrated luminosity delivered by the LHC up to September 2012, $5.1 \mathrm{fb}^{-1}$ at $\sqrt{s}=7 \mathrm{TeV}$ and $12.1 \mathrm{fb}^{-1}$ at $8 \mathrm{TeV}$. The significance of the signal results $6.9 \sigma$. The mass is measured to be $125.8 \pm 0.4$ (stat) \pm 0.4 (syst) $\mathrm{GeV}$. The individual analysis channels are combined and tested in various ways against the predictions of the SM in terms of the coupling structure, observing in all the cases a good agreement. Under the assumption that the observed boson has spin zero, the data disfavour the pseudo-scalar hypothesis $0^{-}$with a $\mathrm{CL}_{s}$ value of $2.4 \%$.
\end{abstract}

\section{Introduction}

On $4^{\text {th }}$ July 2012 the ATLAS [1] and CMS [2] collaborations announced the discovery in the context of the searches for the Standard Model Higgs Boson [3] of a narrow bosonic resonance around $125 \mathrm{GeV}$. For each experiment the combination of the analyses of several decay channels led to the exclusion of the background-only hypothesis at the level of five standard deviations. The discovery was based on a data sample corresponding to the integrated luminosity delivered by the LHC in 2011 $\left(\mathrm{L}=5.1 \mathrm{fb}^{-1}\right.$ at $\left.7 \mathrm{TeV}\right)$ and up to June $2012\left(\mathrm{~L}=5.3 \mathrm{fb}^{-1}\right.$ at 8 $\mathrm{TeV})$. As of September 2012, the available $8 \mathrm{TeV}$ data-set doubled in size, extending to $\mathrm{L}=12.2 \mathrm{fb}^{-1}$. The CMS collaboration updated the results for most of the Higgs analysis channels, strengthening the statistical significance of the signal observed in July. Established the presence of this Higgs particle candidate, its compatibility with the SM predictions needs to be assessed.

Table 1 summarizes the combinations of production and decay modes addressed by the CMS analyses focusing on the low Higgs mass range. For each mode, the integrated luminosity considered so far is displayed. The untagged" category refers to the case where only the Higgs decay products are required, whereas the analyses looking at associated productions demand the presence of additional objects in the event: two forward jets for the $(V B F$ tag) category (Vector Boson Fusion), a leptonic $\mathrm{W}$ or $\mathrm{Z}$ for the VH-tag (Higgs-strahlung) and two top candidates for the $t \bar{t} H$-tag. The properties of the Higgs boson candidate are inferred combining the information provided individually by these channels.

The mass measurement will be described in the Section 3. Once the mass of the Higgs is known, the SM is left with no more free parameters and becomes fully predictive at the electroweak scale. The best estimate for the mass of

\footnotetext{
a e-mail: marco.zanetti@cern.ch
}

Table 1. Synoptic view of the Higgs production (columns) and decay (rows) modes addressed by the CMS analyses. For each entry in the table the integrated luminosity considered in the analysis is reported, the first value referring to the luminosity collected at $\sqrt{s}=7 \mathrm{TeV}$, the second at $\sqrt{s}=8 \mathrm{TeV}$. In the case no number is reported, that combination of production and decay mode has not been addressed.

\begin{tabular}{|c|c|c|c|c|}
\hline & untagged & VBF-tag & VH-tag & $t \bar{t}$ H-tag \\
\hline$H \rightarrow \gamma \gamma$ & $5.1,5.3$ & $5.1,5.3$ & - & - \\
\hline$H \rightarrow Z Z$ & $5.0,12.1$ & - & - & - \\
\hline$H \rightarrow W^{+} W^{-}$ & $4.9,12.1$ & $4.9,12.1$ & $4.9,5.1$ & - \\
\hline$H \rightarrow b \bar{b}$ & - & - & $5.0,12.1$ & $5.0,0$ \\
\hline$H \rightarrow \tau^{-} \tau^{+}$ & - & $4.9,12.1$ & $5.0,0$ & - \\
\hline
\end{tabular}

the new resonance will be then used to probe the coupling structure of the observed signal in Sections 4 and 5.

\section{Model Parameters extraction}

In the following, models parameters (mass, signal or couplings strength, etc.) are evaluated from the scan of the profile likelihood ratio $q(a)$ [4]:

$$
q(a)=-2 \ln \frac{\mathcal{L}\left(\mathrm{obs} \mid s(a)+b, \hat{\theta}_{a}\right)}{\mathcal{L}(\mathrm{obs} \mid s(\hat{a})+b, \hat{\theta})},
$$

The parameters $\hat{a}$ and $\hat{\theta}$ that maximize the likelihood are called the best-fit set. The $68 \%(95 \%) \mathrm{CL}$ on a given parameter of interest $a_{i}$ is evaluated from $q\left(a_{i}\right)=1$ (3.84) with all other unconstrained model parameters treated in the same way as the nuisance parameters. The two dimensional 68\% (95\%) CL contours for pairs of parameters are derived from $q\left(a_{i}, a_{j}\right)=2.3(6)$.

\section{Mass measurement}

Both the di-photon and the four leptons (where for leptons only muons or electrons are meant) final states feature high 


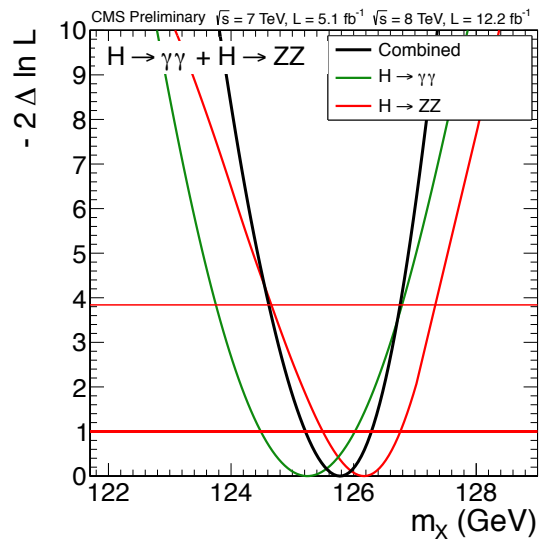

Figure 1. Likelihood scan versus the boson mass $m_{\mathrm{X}}$ for the $\gamma \gamma$ (green) and $4 \ell$ (red) final states separately and for their combination.

resolution for the mass of the parent particle (indicated as $m_{\mathrm{X}}$ henceforth). To extract the value of $m_{\mathrm{X}}$ the untagged and $V B F$-tagged $\mathrm{H} \rightarrow \gamma \gamma$ and $\mathrm{H} \rightarrow \mathrm{ZZ} \rightarrow 4 l$ processes are used, assuming them to entail the same particle but without binding them to the SM expectation. In the fit for the mass, the signal strengths (the factor scaling the signal cross section with respect to the SM prediction) of the three processes are thus taken as independent parameters. Figure 1 shows the scan of the test statistic as a function of the hypothesized mass $m_{\mathrm{X}}$ for the two final states separately and their combination. Crossings of the $q\left(m_{\mathrm{X}}\right)$ curves with the horizontal thick (thin) lines at 1 (3.8) define the $68 \%$ (95\%) confidence level (CL) intervals for the mass of the observed particle. These intervals include both statistical and systematic uncertainties. The $68 \% \mathrm{CL}$ interval is $m_{\mathrm{X}}=125.8 \pm 0.5$.

To evaluate the statistical component of the quoted uncertainty, we performed the fit fixing the nuisance parameters to their best fit value (i.e. without profiling them at each point of the scan). Under the assumption that the total error is given by the sum in quadrature of the statistical and systematic components, we can estimate the latter to be $0.4 \%$.

To assess the dependency of the mass measurement on the SM Higgs boson hypothesis, the fit is repeated using the same channels but constraining instead all production cross sections and branching ratios to the SM predictions as a function of $m_{\mathrm{H}}$. The two results are compatible to better than $0.1 \mathrm{GeV}$, both with respect to the central value and the uncertainties.

\section{Analysis of the coupling structure}

\subsection{Signal strength}

Once determined the mass, the new boson can be confronted with the SM Higgs hypothesis. As a first test, we can assess whether the signal strengths measured by the individual channels are compatible among each other

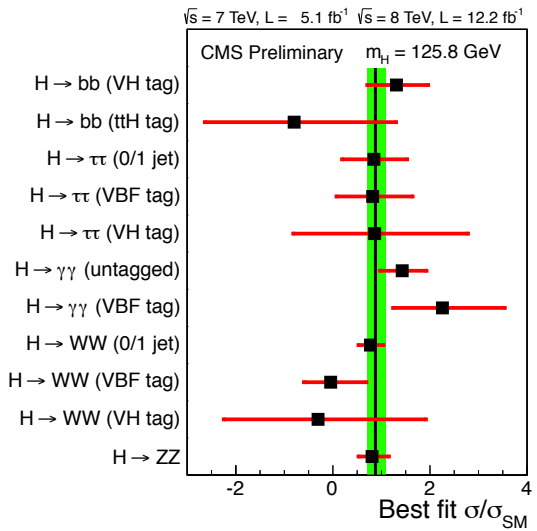

Figure 2. Values of $\sigma / \sigma_{\mathrm{SM}}$ for the combination (solid vertical line), for individual decay modes (point) or sub-combinations of decay modes. The vertical green band shows the overall $\sigma / \sigma_{\mathrm{SM}}$ uncertainty. The horizontal bars indicate the \pm 1 standard deviation uncertainties in the $\sigma / \sigma_{\text {SM }}$ values for the individual modes.

and with the SM expectation. The plot in Figure 2 displays the best fit values of $\sigma / \sigma_{\mathrm{SM}}$ obtained from the set of exclusive channels classified in Tab. 1. The overall compatibility with the SM prediction is very good, none of the modes is departing significantly from the SM expectation $\left(\sigma / \sigma_{\mathrm{SM}}=1\right)$. The combined measurement gives $\sigma / \sigma_{\mathrm{SM}}=0.88 \pm 0.21$, indeed well in agreement with the SM. The overall significance of the signal is 6.9 standard deviations.

It must be noted that the tagging of the production process can suffer from substantial contamination. A relevant example is the di-jet VBF tag that, assuming the SM Higgs cross sections, presents a substantial fraction (20-50\%) of gluon-gluon fusion events. The results shown in Fig. 2 should not be interpreted literally as compatibility tests for pure production mechanisms and decay modes.

\subsection{Physics Framework}

In order to go further in the confrontation with the SM Higgs hypothesis and probing the coupling structure of the new boson, we need a physics framework that allows interpreting the results of the various analyses. CMS follows the proposal formulated in [5], which provides recommendations to seek for (small) deviation from the SM prediction, without hypothesizing any specific phenomenology beyond the SM. A few assumptions have to be taken:

- The signal consists of a single narrow resonance

- The tensor structure corresponds to the one of the SM Higgs sector

- The width of the resonance, $\Gamma_{T}$, is small enough to rely on the zero-width approximation:

$$
\sigma(\mathrm{ii} \rightarrow \mathrm{H} \rightarrow \mathrm{ff}) \sim \frac{\Gamma_{\mathrm{H} \rightarrow \mathrm{ii}} \Gamma_{\mathrm{H} \rightarrow \mathrm{ff}}}{\Gamma_{\mathrm{T}}}
$$

The reason for the second requirement originates from the fact that a different tensor structure would modify some of 
the final state differential distributions the analyses are currently relying on to distinguish the signal from the background. Such assumption is relaxed in Section 5. Equation 2 allows addressing the modifications of the coupling constants regulating the Higgs candidate interactions with the other SM fields:

$$
\sigma(\mathrm{ii} \rightarrow \mathrm{H} \rightarrow \mathrm{ff})=\sigma(\mathrm{ii} \rightarrow \mathrm{H} \rightarrow \mathrm{ff})_{\mathrm{SM}} \frac{\kappa_{i}^{2} \kappa_{f}^{2}}{\kappa_{\mathrm{T}}^{2}}
$$

where the $\kappa$ factors are the modifier of the SM couplings.

The current reach of the Higgs analyses does not allow quantifying meaningfully all the possible degrees of freedom present in the SM. Specific physics models are therefore implemented grouping together subsets of coupling factors with the goal of probing well defined properties of the Higgs sector.

The Higgs can couple to massless gauge bosons via loops. Such processes are particularly relevant at the LHC, the coupling to the gluon driving the main production process and the coupling to the photon leading to one of the most sensitive decay channel. The relative strength factors can either be assumed to be additional free parameters in the fit $\left(\kappa_{\mathrm{g}}\right.$ and $\left.\kappa_{\gamma}\right)$, or the individual contribution from the other SM fields in the loop can be resolved accordingly to the SM predictions. In the latter case the parametric dependency on the concerned strength factors can be derived analytically. As an example the Higgs to gluon coupling can be expressed as:

$\kappa_{\mathrm{g}}^{2}\left(\kappa_{\mathrm{t}}, \kappa_{\mathrm{b}}, m_{\mathrm{H}}\right)=\frac{\kappa_{\mathrm{t}}^{2} \sigma_{\mathrm{ggH}}^{\mathrm{tt}}\left(m_{\mathrm{H}}\right)+\kappa_{\mathrm{b}}^{2} \sigma_{\mathrm{ggH}}^{\mathrm{bb}}\left(m_{\mathrm{H}}\right)+\kappa_{\mathrm{t}} \kappa_{\mathrm{b}} \sigma_{\mathrm{ggH}}^{\mathrm{tb}}\left(m_{\mathrm{H}}\right)}{\sigma_{\mathrm{ggH}}^{\mathrm{tt}}\left(m_{\mathrm{H}}\right)+\sigma_{\mathrm{ggH}}^{\mathrm{bb}}\left(m_{\mathrm{H}}\right)+\sigma_{\mathrm{ggH}}^{\mathrm{tb}}\left(m_{\mathrm{H}}\right)}$

where $\sigma_{\mathrm{ggH}}^{\mathrm{tt}}, \sigma_{\mathrm{ggH}}^{\mathrm{bb}}$ and $\sigma_{\mathrm{ggH}}^{\mathrm{tb}}$ denote respectively the square of the top-quark contribution, the square of the bottomquark contribution and the top-bottom interference computed at NLO QCD accuracy.

The total width cannot be determined at the LHC without making any assumption. The more straightforward approach is to define the total witdh as the sum of the partial widths of the SM Higgs decays:

$$
\kappa_{\mathrm{T}}^{2}=\sum_{\mathrm{i} \in \mathrm{SM}} \kappa_{i}^{2} \mathrm{BR}_{i}
$$

(where the sum extends to the SM particles the Higgs decays into). In the case of undetectable decays (e.g. into a charm quark pair) or for couplings not assessed in the fit, the corresponding contribution is tied to the group the decay products belong to (for example, the coupling to the charm quark is bound to be the same as the top quark in the "fermion sector" model, Section 4.5). In order to probe the possibility of invisible decays due to particles not predicted by the SM, the total width can be parametrized by means of an additional parameter, $\mathrm{BR}_{\mathrm{BSM}}=\frac{\Gamma_{\mathrm{BSM}}}{\Gamma_{\mathrm{T}}}$ :

$$
\kappa_{\mathrm{T}}^{2} \mapsto \frac{\kappa_{\mathrm{T}}^{2}}{1-\mathrm{BR}_{\mathrm{BSM}}}
$$

Unless explicitly stated (as in Sec. 4.6), $\mathrm{BR}_{\mathrm{BSM}}=0$ will always be assumed.

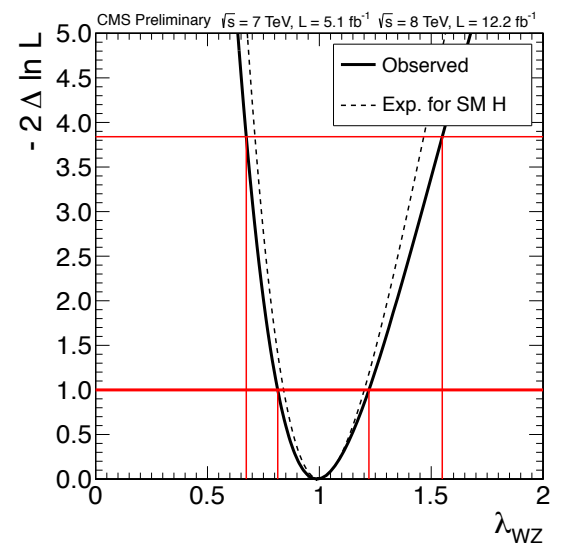

Figure 3. Likelihood scan versus $\lambda_{\mathrm{wZ}}$, the ratio of the couplings to $\mathrm{W}$ and $\mathrm{Z}$ bosons. obtained from the combination of all channels, profiling the coupling to fermions. The solid curve corresponds to the data. The dashed line indicates the expected median results in the presence of the SM Higgs boson. Crossings with the horizontal thick and thin red lines denote the $68 \%$ CL and $95 \% \mathrm{CL}$ intervals.

In the following the physics models exploited for the coupling structure analysis are described and the corresponding results obtained on the available data-set are shown.

\subsection{Custodial Symmetry}

Most of the theoretical implementations of the $\mathrm{SU}(2)_{\mathrm{L}} \times$ $\mathrm{U}(1)_{Y}$ symmetry breaking feature a global unbroken $\mathrm{SU}(2)$ symmetry. One of the consequence of this symmetry, known as "custodial symmetry", is that the coupling strengths of the Higgs to the $\mathrm{W}$ and $\mathrm{Z}$ bosons are linked, in particular the ratio $\lambda_{\mathrm{WZ}}=\kappa_{\mathrm{W}} / \kappa_{\mathrm{Z}}$ cannot deviate from 1 by a sizable amount. To assess this property, the results from each analysis are combined and fit using $\kappa_{\mathrm{F}}, \kappa_{\mathrm{Z}}$ and $\lambda_{\mathrm{WZ}}$ as degrees of freedom. Specifically, each fermion coupling is scaled by the same factor, $\kappa_{\mathrm{F}}$, as well as the coupling to the gluons, whereas the coupling to the photon is resolved at NLO accuracy (similarly to Eq. 4), with the W boson accounting for $\sim 85 \%$ of the amplitude (scaled by $\kappa_{\mathrm{Z}} \lambda_{\mathrm{WZ}}$ ) and the fermions for $\sim 15 \%$.

The plot in Fig. 3 shows the likelihood scan versus $\lambda_{\mathrm{WZ}}$ for the data (solid line) and for the expected median results in the presence of the SM Higgs (dashed line). $\kappa_{\mathrm{F}}$ and $\kappa_{\mathrm{Z}}$ are profiled in the fit together with the other nuisance parameters. The $95 \% \mathrm{CL}$ interval for $\lambda_{\mathrm{WZ}}$ obtained in the combination of all channels is $[0.67,1.55]$ with the data then not disagreeing with the expectation set by the custodial symmetry.

As a further test, the same fit is run but including only the outcome of the untagged $\mathrm{H} \rightarrow \mathrm{ZZ} \rightarrow 41$ and $\mathrm{H} \rightarrow \mathrm{WW} \rightarrow 2 \mathrm{l} 2 v$ analyses. The results are compatible with the full combination with a slighter weaker constrain on the $95 \%$ CL interval, [0.57, 1.65].

In the following models $\lambda_{\mathrm{WZ}}$ will assumed to be one and a unique scale factor $\kappa_{\mathrm{V}}$ will be considered. 


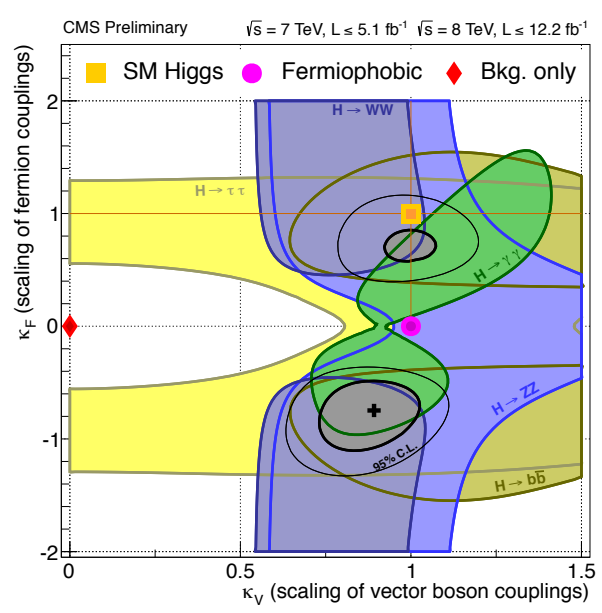

Figure 4. The 68\% CL contours for individual channels (coloured swaths) and for the overall combination (solid line) for the $\left(\kappa_{\mathrm{F}}, \kappa_{\mathrm{V}}\right)$ parameters. The cross indicates the global best-fit values. The thin contour shows the 95\% CL range for the combination. The yellow diamond shows the SM expectation $\left(\kappa_{\mathrm{F}}, \kappa_{\mathrm{V}}\right)$ $=(1,1)$.

\subsection{Weak Vectors and Fermions}

Once verified the consistency of the custodial symmetry prediction, the coupling to the weak vector bosons and to the fermions can simultaneously be examined. The likelihood is scanned in the $\left(\kappa_{\mathrm{F}}, \kappa_{\mathrm{V}}\right)$ plane with the former modifier defined in the same manner as in Section 4.3 and the latter scaling the couplings to $\mathrm{W}$ and $\mathrm{Z}$ bosons.

The results of the fit are shown in the plot of Fig. 4. The contributions of the different decay modes are indicated by the colored contours, whereas the result of the overall combination is identified by the black solid lines. The contours boundaries represent the $95 \%$ confidence region (the gray area represents the $68 \%$ confidence region for the combination). The interference term in the $\mathrm{H} \rightarrow \gamma \gamma$ decay amplitude is the only place where the two parameters $\kappa_{\mathrm{F}}$ and $\kappa_{\mathrm{V}}$ appear as a linear product and therefore the ambiguity on their relative sign can be resolved. The slight excess observed in the di-photon channel is causing the best fit to end up in the $\kappa_{\mathrm{F}}<1$ quadrant. The difference between the global minimum and the local minimum in the $\kappa_{\mathrm{F}}>1$ quadrant is however not statistically significant.

The $95 \%$ CL intervals for $\kappa_{\mathrm{V}}$ and $\kappa_{\mathrm{F}}$ are each obtained from a $1 \mathrm{D}$ scan where the other parameter is fixed to unity, and equal to $[0.78,1.19]$ and $[0.40,1.12]$, respectively.

\subsection{Fermion sector}

In order to probe the fermion sector we exploit a model with three degrees of freedom, one for the scaling of the vectorial couplings, the other two addressing possible asymmetries between fermions types. More specifically we test for the symmetry between lepton and quarks and between fermions with weak isospin $I_{3}=+\frac{1}{2}$ and $I_{3}=-\frac{1}{2}$ (up-type and down-type). In addition to $\kappa_{\mathrm{V}}$ the relevant
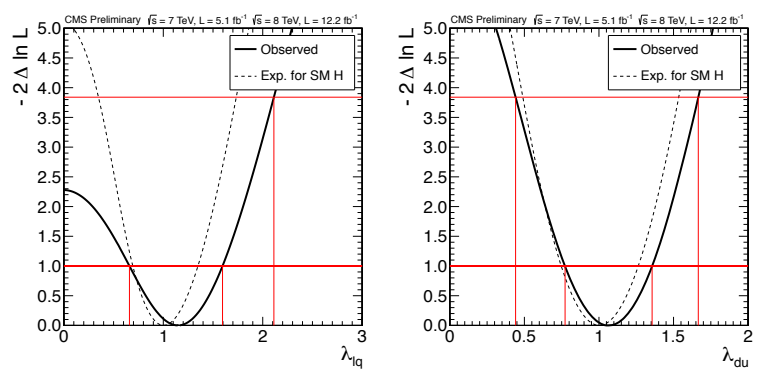

Figure 5. (Left) Likelihood scan versus ratio of couplings to leptons and quarks $\lambda_{l q}$, with the two other free coupling modifiers, $\kappa_{1}$ and $\kappa_{\mathrm{V}}$, profiled together with all other nuisance parameters. (Right) Likelihood scan versus ratio of couplings to down/up fermions $\lambda_{d u}$, with the two other free coupling modifiers, $\kappa_{\mathrm{d}}$ and $\kappa_{\mathrm{V}}$, profiled together with all other nuisance parameters.

parameters for the former model are $\kappa_{\mathrm{q}}$ and $\lambda_{\mathrm{lq}}=\kappa_{\mathrm{l}} / \kappa_{\mathrm{q}}$ and $\kappa_{\mathrm{u}}$ and $\lambda_{\mathrm{du}}=\kappa_{\mathrm{d}} / \kappa_{\mathrm{u}}$ for the latter. This approach is inspired by the class of models with a second Higgs doublet (2HDM), where the presence of additional four Higgs bosons can modify substatially the Yukawa couplings of the SM Higgs.

The plots in Figure 5 display the likelihood scan versus $\lambda_{l q}$ (left) and $\lambda_{d u}$ (right), with the other degrees of freedom profiled together with the nuisance parameters. The relative sign between $\kappa_{1}$ and $\kappa_{\mathrm{q}}$ and between $\kappa_{\mathrm{d}}$ and $\kappa_{\mathrm{u}}$ can hardly be sorted out, the scan is thus performed only in the $\lambda>0$ region (with a good approximation the likelihood behaviour in the negative region is symmetric, both for the SM expectation and for the observed result). The 95\% CL intervals are [0.00,2.11] and [0.45, 1.66] for $\lambda_{l q}$ and $\lambda_{d u}$ respectively, with the SM predictions in both cases well in agreement with the results.

\subsection{Loops and Beyond SM}

The Higgs coupling structure would be modified by new particles not predicted by the SM. In particular the loopmediated couplings ( $\mathrm{gg} \rightarrow \mathrm{H}$ and $\mathrm{H} \rightarrow \gamma \gamma$ ) are expected to be very sensitive to the presence of new states. To spot out BSM effects, the modifiers $\kappa_{\mathrm{g}}$ and $\kappa_{\gamma}$ are used to scale the effective couplings of the Higgs candidate to the gluon and to the photon respectively. The other couplings are fixed in the fit to the SM prediction. The result of the likelihood scan in the $\left(\kappa_{\gamma}, \kappa_{\mathrm{g}}\right)$ plane is shown in the left plot of Fig. 6. The best-fit value is $\left(\kappa_{\gamma}, \kappa_{\mathrm{g}}\right)=(1.43,0.81)$, while the $95 \% \mathrm{CL}$ intervals for each of these couplings separately are [0.98, 1.92] for $\kappa_{\gamma}$ and $[0.55,1.07]$ for $\kappa_{\mathrm{g}}$ (dashed line in the plot).

Effects due to beyond the SM physics can manifest also via invisible Higgs decays. The total width would then be modified as in Eq. 6. In addition to $\kappa_{\mathrm{g}}$ and $\kappa_{\gamma}$ we can then fit for the parameter $\mathrm{BR}_{\mathrm{BSM}}$. The right plot of Fig. 6 displays the result of the likelihood scan versus $\mathrm{BR}_{\mathrm{BSM}}$ with $\kappa_{\mathrm{g}}$ and $\kappa_{\gamma}$ profiled together with the other nuisance parameters. The best fit value results $\mathrm{BR}_{\mathrm{BSM}}=0.0$ whereas the $95 \%$ CL upper bound is 0.62 . 

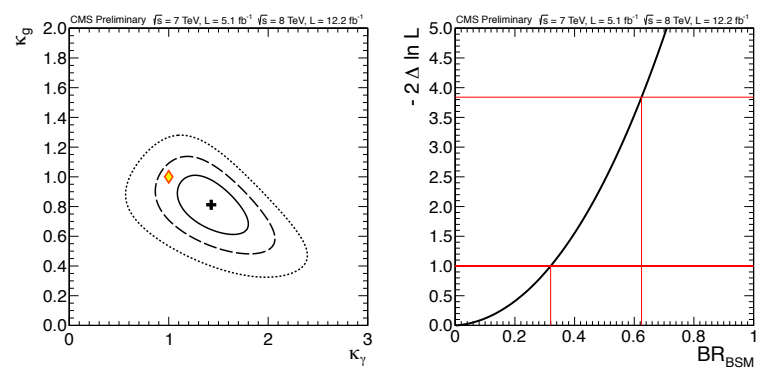

Figure 6. (Left) The 2D likelihood scan for $\kappa_{\mathrm{g}}$ and $\kappa_{\gamma}$ parameters, assuming $\mathrm{BR}_{\mathrm{BSM}}=0$. The cross indicates the best-fit value. The solid, dashed and dotted contours show the $68 \%, 95 \%$ and $99.7 \%$ CL ranges, respectively. The yellow diamond shows the SM point $\left(\kappa_{\gamma}, \kappa_{\mathrm{g}}\right)=(1,1)$. (Right) Likelihood scan versus $\mathrm{BR}_{\mathrm{BSM}}=\Gamma_{\mathrm{BSM}} / \Gamma_{\text {tot }}$. The solid curve is the data and the dashed line indicates the expected median results in the presence of the SM Higgs boson.

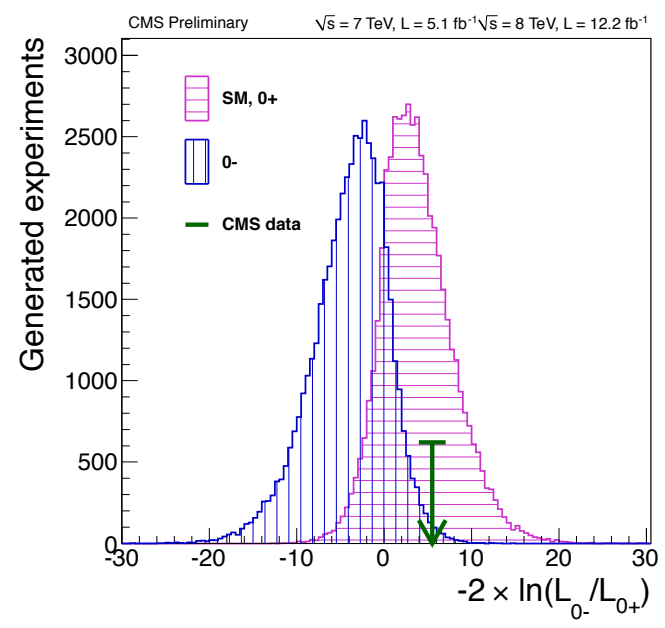

Figure 7. Expected distributions of the test statistic comparing the signal $\mathrm{J}^{\mathrm{P}}$ hypotheses: $0^{-}$vs $0^{+}$. The observed value is indicated by the arrow.

\section{Analysis of the tensor structure}

Modifications to the tensor structure of the SM Higgs sector would reflect in deviations in the expected differential cross sections. The $\mathrm{H} \rightarrow \mathrm{ZZ} \rightarrow 4 l$ decay channel presents a good discriminating power and as of November 2012 is the only process utilized to investigate the charge-parity properties of the signal, more specifically the two $\mathrm{J}^{\mathrm{P}}$ hypotheses $0^{-}$and $0^{+}$are confronted. The state $\mathrm{J}^{\mathrm{P}}=0^{+}$is assumed to be a scalar with a coupling structure to gluons and $\mathrm{Z}$ bosons equal to that of the SM Higgs boson. The exact definitions of the coupling structure of the pseudoscalar state $\mathrm{J}^{\mathrm{P}}=0^{-}$can be found in Ref. [6].

Figure 7 shows the expected distributions of test statistic $q=-2 \ln \left(\mathcal{L}_{0^{-}+\mathrm{bkg}} / \mathcal{L}_{0^{+}+\mathrm{bkg}}\right)$ for background and signal of two types, $0^{-}$and $0^{+}$(SM Higgs boson). The two likelihoods for the alternative hypotheses are maximized independently with respect to the nuisance parameters and the signal strength. The expected distributions are generated with a signal cross-section equal to that of the SM, which is consistent with the observation. The result does not change significantly if the expected distributions are generated with the measured signal strength. The observed value of test statistic deviates from the median expected for the $\mathrm{J}^{\mathrm{P}}=0^{-}$hypothesis by 2.45 standard deviations and is consistent with the median expected for the $\mathrm{J}^{\mathrm{P}}=0^{+}$

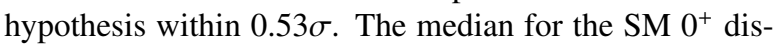
tribution lies 1.93 standard deviations into the tail of the $0^{-}$ distribution, while the median for the $0^{-}$distribution lies 1.99 standard deviations into the tail of the $0^{+}$distribution. We define a $\mathrm{CL}_{\mathrm{s}}$ criterion as the ratio of the probabilities to observe, under the $0^{+}$and $0^{-}$hypotheses, values of the test statistics, equal or larger than the ones observed in the data. Under the assumption that the observed boson has spin zero, the data disfavour the pseudo-scalar hypothesis $0^{-}$with a $\mathrm{CL}_{s}$ value of $2.4 \%$.

\section{Conclusions}

The study of the properties of the signal observed by the CMS experiment in the context of the searches for the SM Higgs boson has been presented. The analyses are based on the data gathered up to September 2012 corresponding to $5.1 \mathrm{fb}^{-1}$ at $7 \mathrm{TeV}$ and $12.1 \mathrm{fb}^{-1}$ at $8 \mathrm{TeV}$. The mass of the new particle is measured from the combination of $\mathrm{ZZ}$ and $\gamma \gamma$ channels to be $125.8 \pm 0.4$ (stat) \pm 0.4 (syst) $\mathrm{GeV}$. The statistical significance of the signal reaches $6.9 \sigma$. The signal strength obtained from the combination of all analyses results to be $\sigma / \sigma_{\mathrm{SM}}=0.88 \pm 0.21$. The coupling structure of the Higgs candidate is probed by comparing the data with the SM predictions. Tests have been performed addressing specific properties of the SM Higgs sector and in all cases a good agreement with the expectations is found. Under the assumption that the observed state has spin zero, the data disfavor the pseudo-scalar hypothesis with a $\mathrm{CL}_{\mathrm{s}}$ value of $2.4 \%$.

\section{References}

[1] ATLAS Collaboration, Phys.Lett. B716 (2012) 1-29

[2] CMS Collaboration, Phys.Lett. B716 (2012) 30-61,

[3] F. Englert and R. Brout, Phys. Rev. Lett. 13 (1964) 321 P.W. Higgs, Phys. Lett. 12 (1964) 132 G. S. Guralnik, C. R. Hagen, and T.W. B. Kibble, Phys. Rev. Lett. 13 (1964) 585

[4] ATLAS and CMS Collaborations, ATL-PHYS-PUB2011-11, CMS NOTE-2011/005

[5] David, A. et al., hep-ph:1209.0040, 2012

[6] CMS Collaboration, hep-ex:1212.6639, 2012 